\title{
Admittedly Simple? The Quest for Clarity in Medicare Claims Data
}

\author{
Jeannine Z Engel, MD ${ }^{1,2 \star}$; Charles FS Locke, MD ${ }^{3,4}$
}

'Department of Medicine, University of Utah School of Medicine, Salt Lake City, Utah; ${ }^{2}$ Compliance Services, University of Utah Health, Salt Lake City, Utah; ${ }^{3}$ Department of Care Coordination/Clinical Resource Management, ${ }^{4}$ Johns Hopkins Hospital, Baltimore, Maryland; ${ }^{4}$ Department of Medicine, Johns Hopkins School of Medicine, Baltimore, Maryland.

very reader of a certain age will recognize this acronym: ADCVANDIML. In simpler times, we "admitted" to a location: medical intensive care unit, bone marrow transplant unit. At some point, admission orders changed from a synonym for "hospitalize" to chart evidence necessary for inpatient payment to the hospital. In the billing and payment world, "inpatient" and "outpatient" hospitalizations are paid at different rates. Observation stays are one type of "outpatient hospitalization," a confusing and contradictory term to physicians and patients alike. In their article published in this month's Journal of Hospital Medicine, Sheehy and colleagues attempt the herculean task of defining a reproducible methodology to identify observation hospital stays using Medicare claims data. ${ }^{1}$ They highlight the complexity of claims data, the variability of revenue codes used, and the probable high frequency of status changes from outpatient observation to inpatient, and vice-versa, during a single hospitalization. They also argue for reform to simplify payment policy for hospitalized patients.

In October 2013, the Center for Medicare and Medicaid Services (CMS) changed the definition of "inpatient" in the Hospital Inpatient Prospective Payment System rule. ${ }^{2}$ This change is known colloquially as the "two-midnight rule" and occurred on the heels of several years of Recovery Audit contractor (RAC) retroactive denials of short-stay inpatient payments to hospitals around the country. These denials appear to have been based solely on the visit status under which a claim was billed, rather than a dispute over the actual medical care delivered. ${ }^{3}$ The RAC audits alleged billions of dollars of improper payment to hospitals and resulted in a log-jam of hundreds of thousands of cases in the federal appeal system. ${ }^{4}$ The two-midnight rule altered the subjective characterization of an inpatient from patient-based (severity of illness) and physician-based (intensity of service) to an objective, time-based payment definition. For the hospital to submit a claim to Medicare Part A, a medical provider with admitting privileges should expect that the patient will need, for medically necessary reasons, a hospitalization that will span at least two midnights of hospital care. Notable exceptions to the rule include patients undergoing a procedure on the Medicare Inpatient Only list and hospital-

*Corresponding Author: Jeannine Z. Engel, MD; E-mail: Jeannine.engel@hsc. utah.edu; Telephone: 801-585-1435.

Received: November 8, 2018; Accepted: December 1, 2018

๑ 2019 Society of Hospital Medicine DOI 10.12788/jhm.3135 izations that include an unplanned mechanical intubation. To receive payment for observation (an outpatient service billed under Part B) the physician must place an observation order in addition to other requirements. At its core, the two-midnight rule is a payment rule, not a patient care rule.

This change in the criteria for an inpatient hospitalization from a subjective to a more objective and measurable timebased criterion might lead us to believe that the process for determining the correct visit status would now be simple. Unfortunately, we are dealing with a messy real-world scenario, where doctors can make different judgments and patients can have an unpredictable hospital course. Physicians are familiar with the issues surrounding the choice of the "correct" admission order. In many hospitals, the Medicare patients in "observation" and those with an "inpatient" order can be on the same floor and even share the same room. From a hospital resource, nurse's, and physician's standpoint, the patients are often indistinguishable. While some facilities have observation units often associated with their emergency departments, the elderly and those patients with certain comorbidities can be excluded from these units based on protocols designed to improve outcomes and patient safety.

Additionally, most patients who spend at least one night in the hospital for medical treatment would not think that they could be an "outpatient." To address this, CMS has produced specific beneficiary information ${ }^{5}$ and now requires hospitals to provide patients with the Medicare outpatient observation notice (MOON) if patients spend more than 24 hours in observation status. ${ }^{6}$ Beneficiaries must sign this notice, but unlike those admitted as inpatients, Medicare observation patients have no appeal rights. Recent articles in the lay press highlight the interplay between observation status, out-of-pocket expenses, and impact on postacute care. ${ }^{7.8}$

Following the implementation of the two-midnight rule, CMS directed the regional Medicare Administrative Contractors to perform audits in every hospital in the country. This has led to system-based processes at most facilities directing the "proper" visit class orders for our patients: direct education to providers, electronic medical record fixes and hard-stops, and real-time communications from the utilization review nurses and staff. These processes, based on a payment rule are burdensome to patients, physicians, and hospital support staff.

It's not surprising to see that the billing of hospital-based observation care is also a quagmire. The methods and results sections of Sheehy et al.'s article reads like a calculus textbook written in a foreign language on first pass, even to an expert. 
Adding to an already complex issue, since October 2013, a hospital's Utilization Review physicians can also "self-deny" Medicare inpatient stays that do not meet the two-midnight rule payment criteria and still bill for most of Part B charges. These cases are sometimes referred to as "Part A to B rebills" and may or may not have been captured in the claims data reported by CMS and reviewed by Sheehy et al. These cases represent another important status change that should be tracked.

There is a multitude of opinions on the pros and cons of observation care as a payment policy, and the data presented

\section{References}

1. Sheehy AM, Shi F, Kind AJH. Identifying observation stays in Medicare data. J Hosp Med. 2019; 114(2):96-100. doi: 10.2788/jhm.3038.

2. Hospital Inpatient Prospective Payment Systems for Acute Care Hospitals and the Long-Term Care; Hospital Prospective Payment System and Fiscal Year 2014 Rates; Quality Reporting Requirements for Specific Providers; Hospital Conditions of Participation; Payment Policies Related to Patient Status; Final Rule. https://www.gpo.gov/fdsys/pkg/FR-2013-08-19/pdf/2013-18956. pdf. Accessed November 1, 2018.

3. Sheehy AM, Locke C, Engel JZ, et al. Recovery audit contractor audits and appeals at three academic medical centers. J Hosp Med. 2015;10(4):212-219. doi: $10.1002 / j h m .2332$.

4. Office of Medicare Hearings and Appeals. Memorandum to OMHA Medicare Appellants. http://www.modernhealthcare.com/assets/pdf/CH92573110. pdf. Accessed November 4, 2018. by Sheehy et al. is further evidence that the line between inpatient and observation hospitalizations remains blurred and mutable. The authors demonstrate the need for a consistent methodology to define observation stays and ultimately to study them using claims-based data. Simplicity may be the answer, but first, we must know what we are doing, then we can have a debate on whether or not it needs to change.

Disclosures: The authors have nothing to disclose.

5. Center for Medicare and Medicaid Services. Are You a Hospital Inpatient or Outpatient? https://www.medicare.gov/sites/default/files/2018-09/11435-AreYou-an-Inpatient-or-Outpatient.pdf. Accessed November 4, 2018

6. Center for Medicare and Medicaid Services. Medicare Outpatient Observation Notice website. https://www.cms.gov/Medicare/Medicare-General-Information/BNI/MOON.html. Accessed November 1, 2018.

7. Kodjak, A. How Medicare's Conflicting Hospitalization Rules Most Me Thousands of Dollars. https://www.npr.org/sections/healthshots/2018/04/20/583338114/how-medicares-conflicting-hospitalization-rules-cost-me-thousands-of-dollars. Accessed November 1, 2018.

8. Schroeder, MO. Have You Really Been Admitted as an Inpatient to the Hospital? https://health.usnews.com/health-care/patient-advice/articles/2018-10-18/have-you-really-been-admitted-as-an-inpatient-to-the-hospital. Accessed November 1, 2018 